\title{
Traveling Wave Solution of the Olver-Rosenau Equation Solved by Dynamics System
}

\author{
Mei Xiong, Longwei Chen $\mathbb{D}^{D}$, and Na Yang \\ College of Statistics and Mathematics, Yunnan University of Finance and Economics, Kunming, Yunnan 650221, China \\ Correspondence should be addressed to Longwei Chen; zz1237@ynufe.edu.cn
}

Received 15 January 2020; Accepted 3 March 2020; Published 20 May 2020

Guest Editor: Praveen Agarwal

Copyright (c) 2020 Mei Xiong et al. This is an open access article distributed under the Creative Commons Attribution License, which permits unrestricted use, distribution, and reproduction in any medium, provided the original work is properly cited.

\begin{abstract}
Olver-Rosenau equations presented by Olver and Rosenau can be rewritten to the dynamic system by the wave transformation. The system is a Hamiltonian system with the first integral, and its phase-space and equilibrium point analysis are given in different parameter spaces in detail. On this basis, we can derive various solutions of the original equation relating these orbits in different phase-space planes, and the theoretical basis of the numerical solution is provided for engineering application and production practice.
\end{abstract}

\section{Introduction}

In recent years, nonlinear partial differential equations (NLPDEs) are more and more extensively used to engineering application and production practice. Because of the difficulty of solving these equations, so many methods have been presented in the last decades. The Camassa-Holm $(\mathrm{CH})$ equation including the cubic term, that is, the Fokas-Olver-Rosenau-Qiao (FORQ) equation,

$$
\sigma_{t}+\frac{1}{2}\left[\left(v^{2} \pm\left(v_{x}\right)^{2}\right) \sigma\right]_{x}=0, \quad \sigma=v \pm\left(v_{x x}\right),
$$

has been studied for a long time and achieved so many results, such as the solution of Cauchy initial value problem [1], Holder continuous [2], the algebro-geometric solutions [3], the Cauchy problem of the generalized equation [4], and the nonuniqueness for the equation [5]. A lot of solving methods for the nonlinear partial differential equation are discussed to be applied in engineering and practice areas, such as the sine-Gordon expansion method [6] and the travelling wave method and its conservation laws [7], and so many examples are in this regard. In this paper, the Olver-Rosenau equation

$$
\sigma_{t}=b v_{x}+\frac{1}{2}\left[\left(v^{2} \pm\left(v_{x}\right)^{2}\right) \sigma\right]_{x}, \quad \sigma=v \pm\left(v_{x x}\right),
$$

was discussed by Olver and Rosenau through a reshuffling procedure of the Hamiltonian operators in 1996, and they found that the equation was changed into the Hamiltonian system by the bitransformation structure of the $\mathrm{mKdV}$ equation [8]. Rosenau believed the nonanalytic solitary waves of the equation in 1997 [9]. Later, the equation was derived in 2013 [10]. The resulting Hamiltonian equations are considered by the dynamical system theory and a phasespace analysis of their singular points. Those results of the study proved that the equations can support double compacton solutions. They found that the new Olver-Rosenau compactons are different from the well-known Rosenau-Hyman compacton and Cooper-Shepard-Sodano compacton.

It was recently introduced by $\mathrm{Li}$ in [11], but Li did not give the solution because of the complexity of the integral. We will give more detailed discussion in this paper. In the second section, we discuss bifurcations and phase portraits of the system in all parameters. In the third section, smooth (or bright) solitary wave solutions of the system and their parametric representations are obtained in detail.

$$
\text { Let } v=\phi(x-c t)=\phi(\zeta) \text {; equation (2) follows from }
$$

$$
-c\left(\phi_{\zeta} \pm \phi_{\zeta \zeta \zeta}\right)=b \phi_{\zeta}+\frac{1}{2}\left[\left(\phi^{2} \pm \phi_{\zeta}^{2}\right)\left(\phi \pm \phi_{\zeta \zeta}\right)\right]_{\zeta}
$$


Integrating once with respect to $\zeta$ and letting $\mathrm{d} \phi / \mathrm{d} \zeta=\omega$, equation (3) can be rewritten as

$$
\frac{\mathrm{d} \omega}{\mathrm{d} \zeta}=-\frac{\phi\left(\phi^{2} \pm \omega^{2}+2(b+c)\right)+k}{\omega^{2} \pm \phi^{2} \pm 2 c},
$$

which has the first integral

$$
h(\phi, \omega)=\frac{1}{4}\left(\phi^{2} \pm \omega^{2}\right)^{2}+(b+c) \phi^{2} \pm c \omega^{2}+k \phi,
$$

where $k$ is an integral constant.

Without loss of generality, we consider the case + instead of “ \pm ." Equation (5) is simplified as the dynamic system

$$
\begin{aligned}
& \frac{\mathrm{d} \phi}{\mathrm{d} \zeta}=\omega, \\
& \frac{\mathrm{d} \omega}{\mathrm{d} \zeta}=-\frac{\phi\left(\phi^{2}+\omega^{2}+2(b+c)\right)+k}{\omega^{2}+\phi^{2}+2 c},
\end{aligned}
$$

with the Hamiltonian

$$
h(\phi, \omega)=\frac{1}{4}\left(\phi^{2}+\omega^{2}\right)^{2}+(b+c) \phi^{2}+c \omega^{2}+k \phi .
$$

Obviously, system (6) is a planar dynamical system with three parameters depended on the parameter group $(b, c$, and $k)$. All possible phase portraits of $(6)$ in the $(\phi, \omega)$ phase are discussed under the conditions of the different parameter group $(b, c$, and $k)$.

We notice that the right-hand side of the second equation in (6) is not continuous, while $\omega^{2}+\phi^{2}+2 c=0$. On the circle line, $\omega^{2}+\phi^{2}=-2 c$, in the phase plane $(\phi, \omega), \phi_{\zeta \zeta}$ is not well defined. This implies that differential system (6) could have traveling wave solutions with nonsmoothness.

\section{Phase Portraits of the System}

Imposing the transformation $\mathrm{d} \zeta=\left(\omega^{2}+\phi^{2}+2 c\right) \mathrm{d} \tau$, when $\omega^{2}+\phi^{2}+2 c \neq 0$, equation (6) leads to associated regular system

$$
\left\{\begin{array}{l}
\frac{\mathrm{d} \phi}{\mathrm{d} \tau}=\omega\left(\omega^{2}+\phi^{2}+2 c\right), \\
\frac{\mathrm{d} \omega}{\mathrm{d} \tau}=-\phi\left(\phi^{2}+\omega^{2}+2(b+c)\right)-k .
\end{array}\right.
$$

This system has the same first integral as equation (6). Apparently, the singular curve $\omega^{2}+\phi^{2}+2 c=0$ is related to the singular solution of equation (8). Near the circumference, the variable $\tau$ is a fast variable, while the variable $\zeta$ is a slow variable in the sense of the geometric singular perturbation theory.

In order to find the equilibrium points of system (8), let $f(\phi)=\phi\left(\phi^{2}+2 c+2 b\right)+k$ and $f^{\prime}(\phi)=0$; we obtain $\phi_{1}=$ $-\sqrt{(-2 / 3)(b+c)}$ and $\phi_{2}=-\sqrt{(-2 / 3)(b+c)}$ if $(b+c)<0$. The zero points of $f(\phi)$ are estimated by the monotonicity of the function $\phi$ based on zero points of the derivative function $f^{\prime}(\phi)$. Let $A=-6(b+c), B=-9 k$, and $C=(2 c+2 b)^{2}$; then, the discriminant $S=B^{2}-4 A C$ of the cubic polynomial $f(\phi)=0$ is just that $S=81 g^{2}+96(b+c)^{3}$. It is easy to see that, for given $b$ and $c$ when $k^{2}<k_{1}^{2}=(-32 / 27)(b+c)^{3}$, we have $S<0$. It follows that there exist three simple real roots $\phi_{31}, \phi_{32}$, and $\phi_{33}$ of $f(\phi)$ satisfying $\phi_{31}<\phi_{1}<\phi_{32}<\phi_{2}<\phi_{33}$.

If $k^{2}=k_{1}^{2}$, there exist two simple real roots $\phi_{21}$ and $\phi_{22}$ of $f(\phi)$ satisfying $\phi_{21}=\phi_{1}<\phi_{2}<\phi_{22}$

If $k^{2}>k_{1}^{2}$, there exists one simple real root $\phi_{11}$

If $A=B$, there exists one simple real root $\phi=0$

On the singular circle line, there exist two equilibrium points $S_{\mp}=\left(-k /(2 b), \mp Y_{s}\right)$ of system (8) with $Y_{s}=\sqrt{-k^{2} /\left(4 b^{2}\right)-2 c}$ if $k^{2} /\left(4 b^{2}\right)+2 c<0$.

Let $M\left(\phi_{j}, y_{j}\right)$ be the Jacobi matrix of system (8) at an equilibrium point $E_{j}\left(\phi_{j}, y_{j}\right)$; we have

$$
\begin{array}{r}
J\left(\phi_{j}, 0\right)=\operatorname{det} M\left(\phi_{j}, 0\right)=3 \phi^{4}+2(4 c+b) \phi^{2}+4 b c+4 c^{2}, \\
J\left(-\frac{k}{(2 b)}, \mp Y_{s}\right)=\operatorname{det} M\left(-\frac{k}{(2 b)}, \mp Y_{s}\right)=-\left(\frac{2 k^{2}}{b^{2}}+4 b\right) Y_{s}^{2} .
\end{array}
$$

By the theory of planar dynamical systems, for an equilibrium point of a planar integrable system, if $J<0$, then the equilibrium point is a saddle point; if $J>0$ and $(\operatorname{trace} M)^{2}-4 J<0(>0)$, then it is a center point (a node point); and if $J=0$ and the Poincare index of the equilibrium point is 0 , then this equilibrium point is cusped (see [12]).

Let $h_{i}=H\left(\phi_{i}, 0\right)$ and $h_{s}=H\left(-k /(2 b), \mp Y_{s}\right)$, where $H$ comes from equation (5).

(1) If $S=0$, there exist two simple real roots, and then $k^{2}=k_{1}^{2}=(-32 / 27)(b+c)^{3}, \phi_{21}=(3 k) /(2(b+c)), \phi_{22}$ $=-(3 k) /(4(b+c))$.

(i) When $b>0$ and $-4 b<c<-b,\left(\phi_{21}, 0\right)$ is a saddle point. $\left(\phi_{22}, 0\right)$ is the high-order singular point, see Figure 1.

(ii) When $c<-4 b<0$ or $b<0, c<-b,\left(\phi_{21}, 0\right)$ is a center point. $\left(\phi_{22}, 0\right)$ is the high-order singular point, see Figures 2 and 3.

(2) If $A=B$, there exists only one simple real root $\phi=0$, and then $k=(2 / 3)(b+c)$.

(i) When $b>0,-b<c<0$ or $b<0$, and $0<c<-b$, $(0,0)$ is a saddle point from the high-order singular point, see Figures 4 and 5 .

(ii) When $b>0, c>0$ (Figure 6) or $b>0, c<-b$ (Figure 7 ) or $b<0, c>-b$ (Figure 8 ) or $b<0$, $c<0$ (Figure 9), $(0,0)$ is a center point from the high-order singular point.

(3) If $S<0$, there exist three simple real roots, and then $k^{2}<k_{1}^{2}=(-32 / 27)(b+c)^{3}, \phi_{31}=(-2 / 3) \sqrt{-6(b+c)}$ $\cos (\theta / 3), \phi_{32}=(1 / 3) \sqrt{-6(b+c)}(\cos (\theta / 3)+\sqrt{3} \sin$ $(\theta / 3))$, and $\phi_{33}=(1 / 3) \sqrt{-6(b+c)}(\cos (\theta / 3)-\sqrt{3}$ $\sin (\theta / 3))$, where $\theta=\arccos T, \quad T=(27 k) /(2(-6$ $\left.(b+c))^{3 / 2}\right)$. 


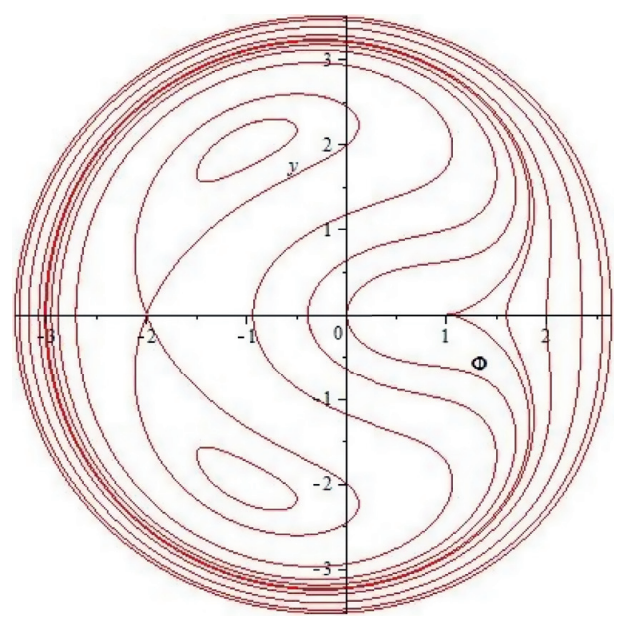

FIgURE 1: One is a saddle point, and another is a high-order singular point $(b>0$ and $-4 b<c<-b)$.

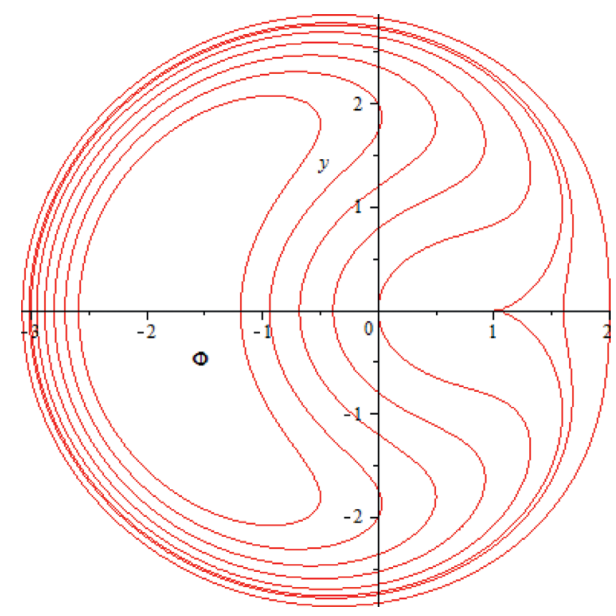

FIgURE 2: One is a center point, and another is a high-order singular point $(c<-4 b<0)$.



FIgURE 3: One is a center point, and another is a high-order singular point $(b<0, c<-b)$.

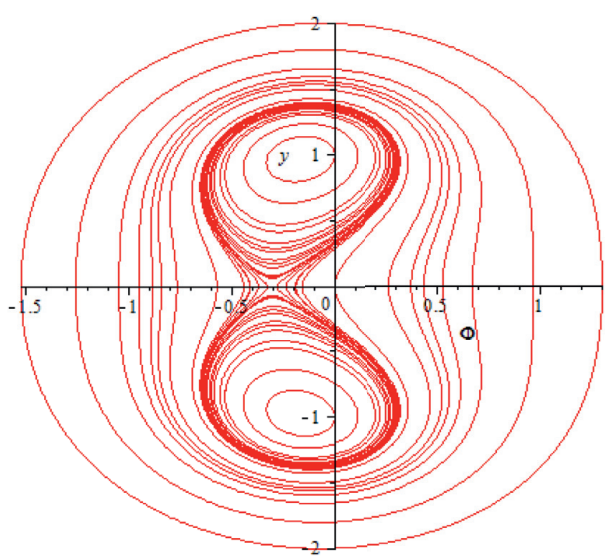

FIgURE 4: $(0,0)$ is a saddle point from the high-order singular point $(b>0,-b<c<0)$.

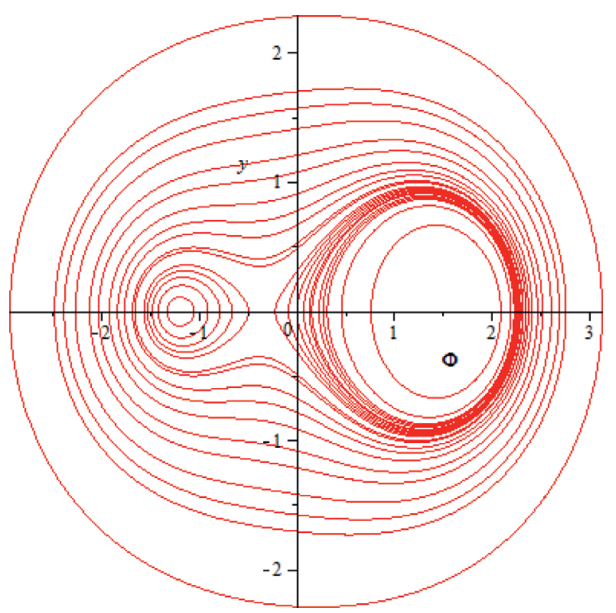

FIgURE 5: $(0,0)$ is a saddle point from the high-order singular point $(b<0,0<c<-b)$.

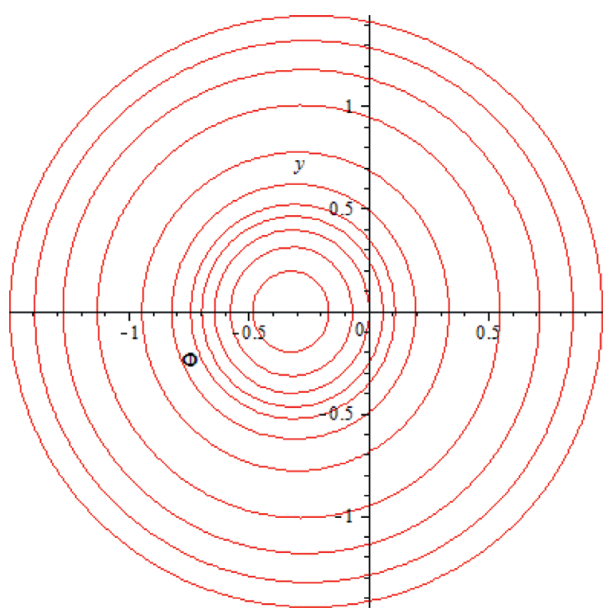

FIgURE 6: $(0,0)$ is a center point from the high-order singular point $(b>0, c>0)$. 


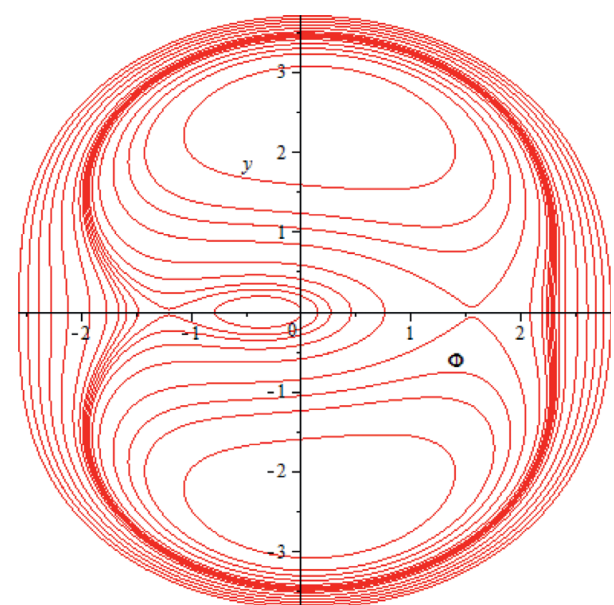

FIgURE 7: $(0,0)$ is a center point from the high-order singular point $(b>0, c<-b)$.

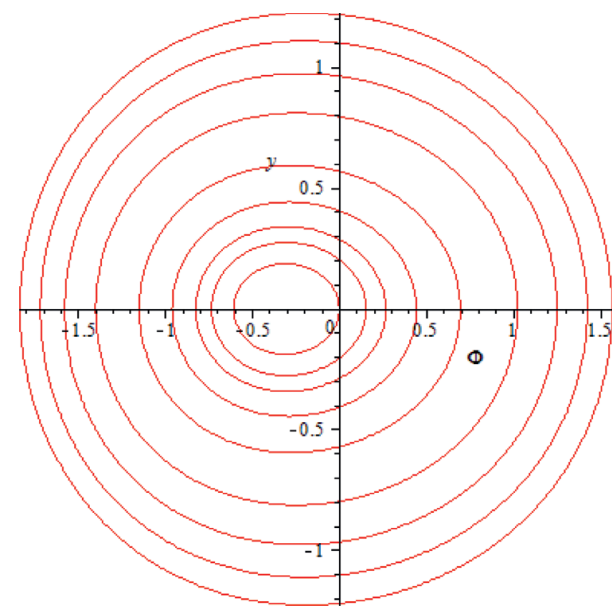

FIGURE 8: $(0,0)$ is a center point from the high-order singular point $(b<0,-b<c)$.

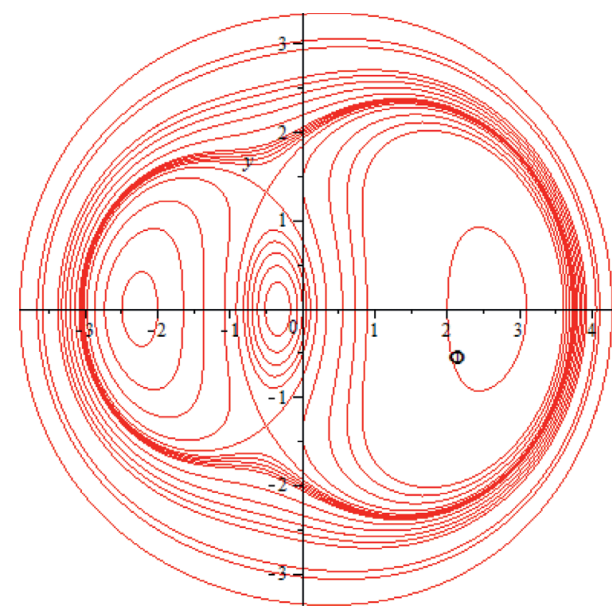

FIGURE 9: $(0,0)$ is a center point from the high-order singular point $(b<0,0>c)$. (i) When $b+c<0, b>2 c,\left(\phi_{31}, 0\right),\left(\phi_{32}, 0\right)$, and $\left(\phi_{33}, 0\right)$ are centers. $\left(-k /(2 b), \mp Y_{s}\right)$ are saddle points, see Figure 10.

(ii) When $b+c<0, b<2 c,\left(\phi_{31}, 0\right),\left(\phi_{32}, 0\right)$, and $\left(\phi_{33}, 0\right)$ are centers. $\left(-k /(2 b), \mp Y_{s}\right)$ are saddle points, see Figure 11.

(4) If $S>0$, there exists one simple real root, and then $k^{2}>k_{1}^{2}=(-32 / 27)(b+c)^{3}$ and $\phi_{11}=(-1 / 3)\left(Y_{1}^{1 / 3}\right.$ $\left.+Y_{2}^{1 / 3}\right)$, where $Y_{1}=(3 / 2)\left(9 k+\sqrt{81 k^{2}+96(b+c)^{3}}\right)$ and $Y_{2}=3 / 2\left(9 k-\sqrt{81 k^{2}+96(b+c)^{3}}\right)$.

(i) When $b<0, c<0,\left(\phi_{11}, 0\right)$ is a center, see Figure 12 .

(ii) When $b>0, c>0,\left(\phi_{11}, 0\right)$ is also a center, see Figure 13.

We obtain four class figures totally including 13 phase portraits under the conditions of different parameters. An orbit in a figure is related to Hamiltonian $h$ of equation (7). An orbit derives a class solution of system (6), and at the same time, it is appropriative to the original partial differential equation. In the next section, we mainly consider how to solve system (6).

\section{Smooth (Bright) Solitary Wave Solutions and Their Parametric Representations}

As we know, system (6) for a fixed integral constant $h$ has an explicit solution. However, the scope of value $h$ is calculated by different orbits corresponding to different parameter conditions in the phase portraits from Figures 1 to 13.

$$
\begin{aligned}
& \omega^{2}=2 \sqrt{h+c^{2}-b \phi^{2}-k \phi}-\left(\phi^{2}+2 c\right), \\
& \frac{\mathrm{d} \phi}{\mathrm{d} \zeta}=\omega=\sqrt{2 \sqrt{h+c^{2}-b \phi^{2}-k \phi}-\left(\phi^{2}+2 c\right)} .
\end{aligned}
$$

To write this equation as integral form,

$$
\int \frac{\mathrm{d} \phi}{\sqrt{2 \sqrt{h+c^{2}-b \phi^{2}-k \phi}-\left(\phi^{2}+2 c\right)}}=\int d \zeta .
$$

As it is very difficult to solve the left integral directly, we believe that square root $F=h+c^{2}-b \phi^{2}-k \phi$ plays a critical role in our discussion. If $F$ is a perfect square of the function $\phi$, we can obtain analytical solution because of the simplicity of the integral. If $F$ is a complete square, then the algebraic expression in the first root in the above formula becomes rational, and the integration is relatively easy solution.

\subsection{Analytical Solution. Supporting that}

$$
F=h+c^{2}-b \phi^{2}-k \phi=-b\left(\phi+\frac{k}{2 b}\right)^{2}
$$

If we want to remove the second root sign of equation (11), we must make $\mathrm{F}$ to be complete square, and the necessary and sufficient condition for $\mathrm{F}$ to be completely square is also given: 


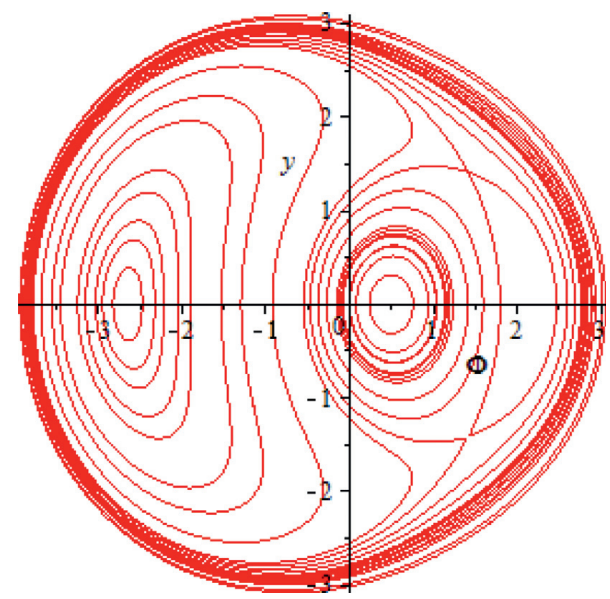

FIGURE 10: Three singular points are centers, and another is saddle $(b+c<0, b>2 c)$.

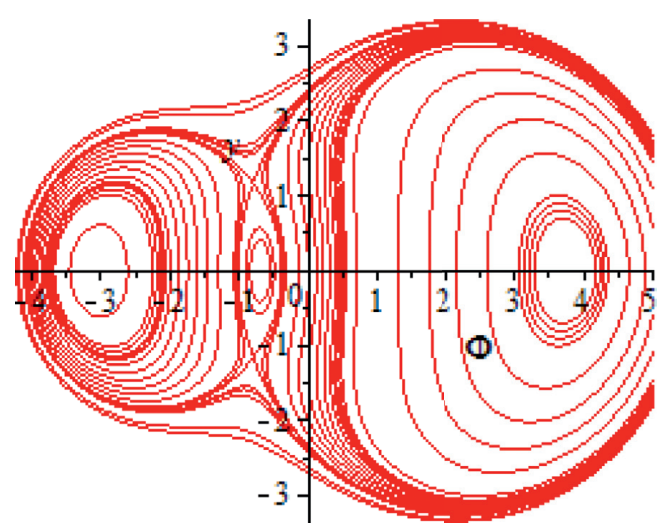

FIGURE 11: Three singular points are centers, and another is saddle $(b+c<0, b<2 c)$.

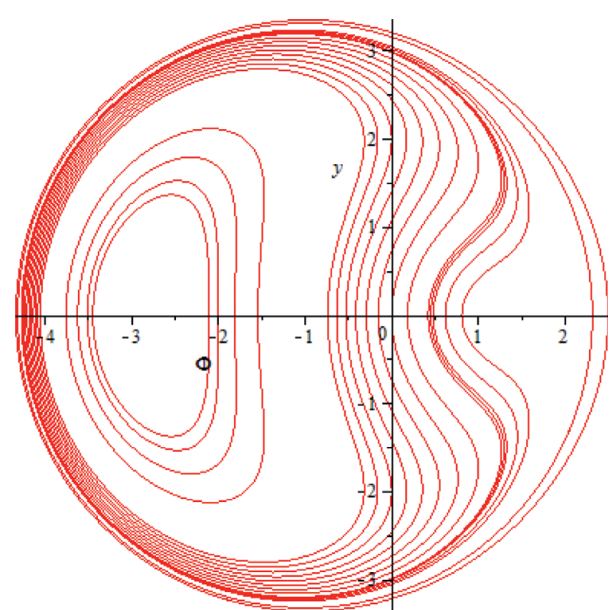

FIGURE $12: b<0, c<0$, and $\left(\phi_{11}, 0\right)$ is a center.

$$
\int \frac{\mathrm{d} \phi}{\sqrt{D-(\phi-\sqrt{-b})^{2}}}=\int \mathrm{d} \zeta
$$

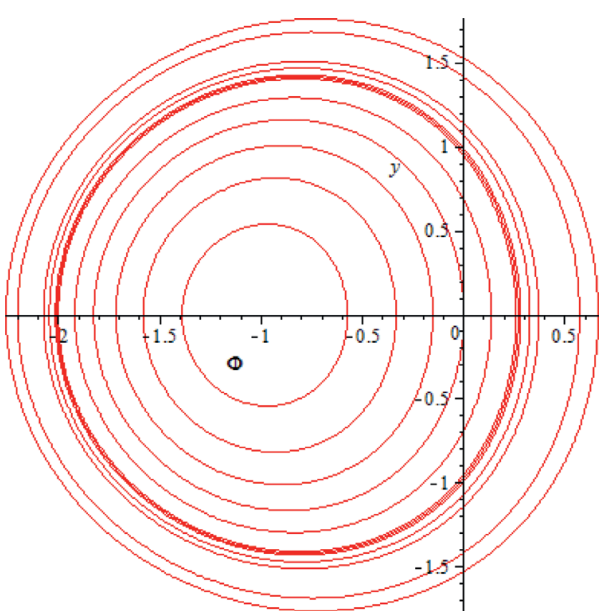

FIgURE 13: $b>0, c>0$, and $\left(\phi_{11}, 0\right)$ is also a center.

where $D=b^{2}+2 c \pm 2 \sqrt{h+c^{2}} \geq 0$ and $-D+\sqrt{-b}<\phi<D$ $+\sqrt{-b}, b<0$. After integrating, we obtain ( $C$ is an integral constant)

$$
\frac{\phi-\sqrt{-b}}{\sqrt{D-(\phi-\sqrt{-b})^{2}}}=\tan (x-c * t+C) .
$$

3.2. Approximate Solution. If $F=\sqrt{h+c^{2}-b \phi^{2}-k \phi}$ is not a rational function, the integral of equation (11) is very difficult to be calculated directly. If $\mathrm{F}$ cannot be expressed as a complete square, we can only carry out Taylor expansion of F; otherwise, equation (13) cannot calculate the integral, and the solution of the original equation cannot be obtained. While we need to approximate nonlinear function, our right choice is the Taylor display:

$$
\begin{aligned}
(1+x)^{\alpha}= & 1+\alpha x+\frac{\alpha(\alpha-1)}{2 !} x^{2}+\cdots \\
& +\frac{\alpha(\alpha-1 \cdots(\alpha-n+1))}{n !}+R_{n}(x),
\end{aligned}
$$

where the remainder

$$
\begin{aligned}
R_{n}(x) & =\frac{\alpha(\alpha-1) \cdots(\alpha-n)}{(n+1) !}(1+\theta x)^{\alpha-n+1}(0<\theta<1), \\
F & =\sqrt{h+c^{2}-b \phi^{2}-k \phi}=\left(h+\frac{k^{2}}{4 b}-b\left(\phi+\frac{k}{2 b}\right)^{2}\right)^{1 / 2} \\
& =\left(\frac{4 b\left(h+c^{2}\right)+k^{2}}{4 b}-b\left(\phi+\frac{k}{2 b}\right)^{2}\right)^{1 / 2} \\
& =\frac{1}{2} \sqrt{\frac{4 b\left(h+c^{2}\right)+k^{2}}{b}}\left(1-\frac{4 b^{2}}{4 b h+4 b c^{2}+k^{2}}\left(\phi+\frac{k}{2 b}\right)^{2}\right)^{1 / 2} .
\end{aligned}
$$


Let $4 b^{2} /\left(4 b\left(h+c^{2}\right)+k^{2}\right)=A$; Taylor expansion is

$$
\begin{aligned}
\sqrt{h+c^{2}-b \phi^{2}-k \phi} & =\sqrt{\frac{b}{A}}\left(1-A\left(\phi+\frac{k}{2 b}\right)^{2}\right)^{1 / 2} \\
& =\sqrt{\frac{b}{A}}\left[1-\frac{1}{2} A\left(\phi+\frac{k}{2 b}\right)^{2}+R_{2}(x)\right],
\end{aligned}
$$

generally satisfying $\left|A(\phi+(k / 2 b))^{2}\right|<1$,

$$
\begin{aligned}
R_{2}(x)= & \frac{1 / 2(1 / 2-t 1)}{2}\left(1+\theta A\left(\phi+\frac{k}{2 b}\right)^{2}\right)^{1 / 2} \\
& \cdot \sqrt{h+c^{2}-b \phi^{2}-k \phi} \\
\approx & \sqrt{\frac{b}{A}}\left[1-\frac{1}{2} A\left(\phi+\frac{k}{2 b}\right)^{2}\right] .
\end{aligned}
$$

$$
\begin{aligned}
& \text { Error }|d| \leq(1 / 8)(1+A \theta(\phi+(k / 2 b)))^{1 / 2} . \\
& \qquad \frac{\mathrm{d} \phi}{\mathrm{d} \zeta}=\sqrt{2 \sqrt{\frac{b}{A}}-\sqrt{b A}\left(\phi+\frac{k}{2 b}\right)^{2}-\left(\phi^{2}+2 c\right)},
\end{aligned}
$$

$$
\int \frac{\mathrm{d} \phi}{\sqrt{-\left(\sqrt{b A}\left(k^{2} / 4 b^{2}\right)+1\right) \phi^{2}-\sqrt{(A / b) k \phi-\left(k^{2} / 4 b^{2}\right)+2 \sqrt{(b / A)}+2 c=\mathrm{d} \zeta}}}
$$

Let $A_{1}=-\left(\sqrt{b A}\left(k^{2} / 4 b^{2}\right)+1\right), \quad B_{1}=-\sqrt{(A / k)} k$, and $C_{1}=-\left(k^{2} / 4 b^{2}\right)+2 \sqrt{b / A}+2 c$; then the above equation of the integral becomes

$$
\int \frac{\mathrm{d} \phi}{\sqrt{A_{1} \phi^{2}+B_{1} \phi+C_{1}}}=\mathrm{d} \zeta
$$

Approximation solution of system (6) is

$$
\ln \left(\frac{1}{2} B_{1}+A_{1} \phi\right)+\sqrt{A_{1} \phi^{2}+B_{1} \phi+C_{1}}=\sqrt{A_{1}} *(x-c * t) .
$$

$v=\phi(x-c * t)$ is the approximation solution of the original equation.

If the order of Taylor expansion of $\mathrm{F}$ is higher, the accuracy of the solution will be higher.

\section{Conclusion}

We obtain some exact solutions and some approximation solutions of the Olver-Rosenau equation by the dynamic system method. The original equation considered by the first integral method and its phase-space analysis and equilibrium points are calculated under different parameter conditions. We can derive various solutions of the original equation relating these orbits in different phase-space planes. Nevertheless, as there are some troubles in the course of the calculation of these solutions because of the integral complexity, we need to find some numerical methods for the equation. The precision differs from the variable order of Taylor expansion.

\section{Data Availability}

This paper is mainly theoretical derivation and calculation, it does not involve data and its right to use.

\section{Conflicts of Interest}

The authors declare that they have no conflicts of interest.

\section{References}

[1] A. Alexandrou Himonas and D. Mantzavinos, "The Cauchy problem for the Fokas-Olver-Rosenau-Qiao equation," Nonlinear Analysis: Theory, Methods \& Applications, vol. 95, no. 1, pp. 499-529, 2014.

[2] A. Alexandrou Himonas and D. mantzavinos, "Holder continuity for the Fokas-Olver-Rosenau-Qiao equation," Journal of Nonlinear Science, vol. 24, no. 6, pp. 1105-1124, 2014.

[3] Y. Hou, E. Fan, and Z. Qiao, "The algebro-geometric solutions for the Fokas-Olver-Rosenau-Qiao (FORQ) hierarchy," Journal of Geometry and Physics, vol. 117, no. 7, pp. 105-133, 2017.

[4] M. Yang, Y. Li, and Y. Zhao, "On the Cauchy problem of generalized Fokas-Olver-Resenau-Qiao equation," Applied Analysis, vol. 8, pp. 1-23, 2017.

[5] A. Alexandrou Himonas and H. Curtis, "Non-uniqueness for the Fokas-Olver-Rosenau-Qiao equation," Journal of Mathematical Analysis and Applications, vol. 470, no. 2, pp. 647658, 2019.

[6] H. Mehmet Baskonus, H. Bulut, and T. A. Sulaiman, "New complex hyperbolic structures to the Lonngren-wave 
equation by using Sine-Gordon expansion method," Applied Mathematics and Nonlinear Sciences, vol. 4, no. 1, pp. 129-138, 2019.

[7] C. M. Khalique and I. E. Mhlanga, "Travelling waves and conservation laws of a $(2+1)$-dimensional coupling system with Korteweg-de Vries equation," Applied Mathematics and Nonlinear Sciences, vol. 3, no. 1, pp. 241-254, 2018.

[8] P. J. Olver and P. Rosenau, "Double compactons in the OlverRosenau eqution," Physics Letters A, vol. 53, pp. 123-128, 1996.

[9] P. Rosenau, "On nonanalytic solitary waves formed by a nonlinear dispersion," Physics Letters A, vol. 230, no. 5-6, pp. 305-318, 1997.

[10] A. Chen and S. Wen, "Double compactons in the OlverRosenau equation," Pramana, vol. 80, no. 3, pp. 471-478, 2013.

[11] J. Li, "Theory of dynamical system," in Singular Nonlinear Travelling Wave Equation: Bifurcations and Exact Solutions, p. 20, Science Press, Beijing, China, 2013.

[12] J. Li and H. Dai, "Bafucation of dynamical system," in On the Study of Singular Nonlinear Travelling Wave Equations: Dynamical Approach, pp. 18-25, Science press, Beijing, China, 2007. 